Pacific

Journal of

Mathematics

NORMAL FAMILIES OF HOLOMORPHIC MAPPINGS INTO COMPLEX PROJECTIVE SPACE CONCERNING SHARED HYPERPLANES

LiU Yang, Caiyun Fang and Xuecheng Pang

Volume 272 No. 1

November 2014 


\title{
NORMAL FAMILIES OF HOLOMORPHIC MAPPINGS INTO COMPLEX PROJECTIVE SPACE CONCERNING SHARED HYPERPLANES
}

\author{
Liu Yang, Caiyun Fang and Xuecheng Pang
}

\begin{abstract}
We prove new criteria for normality for holomorphic mappings into the complex projective space using the generalized Zalcman lemma. This improves previous results in one complex variable. An example is included to complement our theory.
\end{abstract}

\section{Introduction}

Recall that a family $\mathscr{F}$ of meromorphic functions on a plane domain $D \subset \mathbb{C}$ is normal on $D$ if every sequence in $\mathscr{F}$ contains a subsequence that converges uniformly on $D$ (with respect to the spherical metric) to a meromorphic function or to $\infty$.

The following Picard-type theorem is a consequence of the second main theorem of value distribution theory.

Theorem A [Bergweiler 2006, pp. 78-80] . Let $f$ be a meromorphic function on the complex plane $\mathbb{C}$. If there exist three mutually distinct points $a_{1}, a_{2}$ and $a_{3}$ on the Riemann sphere such that $f(z)-a_{j}($ for $j=1,2,3)$ has no zero on the complex plane then $f(z)$ is a constant.

A heuristic principle, bearing Bloch's name and playing an important role in the theory of normal families, says that if the only meromorphic function with a certain property are constant, then a family of meromorphic functions in a plane domain possessing this property is likely to be normal [Bergweiler 2006, pp. 78-80]. For example, the Montel-type theorem associated with Theorem A is true:

Theorem B [Bergweiler 2006, pp. 78-80]. Let $\mathscr{F}$ be a family of meromorphic functions on a plane domain D. Suppose that there exist three mutually distinct points $a_{1}, a_{2}$ and $a_{3}$ on the Riemann sphere such that $f(z)-a_{j}($ for $j=1,2,3)$ has no zero on $D$ for each $f \in \mathscr{F}$. Then $\mathscr{F}_{F}$ is a normal family on $D$.

We say that two meromorphic functions $f$ and $g$ on a domain $D$ share the value $a\left(a=\infty\right.$ is allowed) if $f^{-1}(a)=g^{-1}(a)$ as sets (ignoring multiplicities). There

Supported by National Natural Science Foundation of China (Grant No. 11261029 and 11371139). MSC2010: primary 32A17, 32H25; secondary 30D35, 30D45.

Keywords: normal family, holomorphic mapping, complex projective space. 
are many results concerning this notion in value distribution theory, such as R. Nevanlinna's famous theorem [1926] that two meromorphic functions on the complex plane sharing five distinct values coincide identically. (The number 5 cannot be reduced, as the pair $e^{z}, e^{-z}$, with shared values $0,1,-1, \infty$, demonstrates; but Nevanlinna [1926] also showed that if four values are shared and the multiplicities with which these each of these values is taken are the same for the two functions, the two functions differ only by a Möbius transformation. The condition that the multiplicities are the same cannot be relaxed; see [Gundersen 1979].)

More generally, the maximum modulus principle and Montel's theorem yield this extension of Theorem B:

Theorem C. Let $\mathscr{F}$ be a family of meromorphic functions on a plane domain D. Suppose that there exist three mutually distinct points $a_{1}, a_{2}$ and $a_{3}$ on the Riemann sphere such that for each $f, g \in \mathscr{F}, f$ and $g$ share $a_{j}($ for $j=1,2,3)$ on $D$. Then F is normal on $D$.

The following question arises naturally from Theorem C. Suppose two families of meromorphic functions share some values $a_{j}$. If one is normal, is the other normal? Recently the problem was solved by Pang and Liu, who showed that if two families of meromorphic functions share four values, the normality of one family implies the normality of the other. They also gave a counterexample to show that the number 4 is sharp.

Theorem D [Liu et al. 2013]. Let $\mathscr{F}$ and 9 be two families of meromorphic functions on a plane domain $D$. Suppose that there exist four mutually distinct points $a_{1}, a_{2}, a_{3}$ and $a_{4}$ on the Riemann sphere such that for each $f \in \mathscr{F}$, there exists $g \in \mathscr{G}_{\text {such }}$ that $f$ and $g$ share $a_{j}$ for $j=1, \ldots, 4$ on $D$. If $\varphi$ is normal on $D$, then $\mathscr{F}$ is also normal on $D$.

The classical Zalcman lemma plays a central role in normal family theory of one complex variable. On the other hand, the study of normal families for holomorphic mappings was initiated by H. Wu in his well-known paper in Acta Math [1967]. Much attention has been given to find the correct generalization of Zalcman's result to several complex variables. In this paper we prove some new normality criteria for holomorphic mappings from plane domains into $\mathbb{P}^{s}(\mathbb{C})$ using the generalized Zalcman lemma. An example will be included to complement our theory.

\section{Basic notions and main results}

Basic notions. We start with relevant definitions. For details see [Mai et al. 2005; Shabat 1985, pp. 99-106; Ru 2001, pp. 99-102].

Let $\mathbb{P}^{s}(\mathbb{C})$ be a complex $s$-dimensional projective space and $\rho: \mathbb{C}^{s+1} \backslash\{0\} \rightarrow \mathbb{P}^{s}(\mathbb{C})$ be the standard projective mapping. A subset $H$ of $\mathbb{P}^{s}(\mathbb{C})$ is called a hyperplane if 
there is a $s$-dimensional linear subspace $\widetilde{H}$ of $\mathbb{C}^{s+1}$ such that

$$
\rho(\widetilde{H}-\{0\})=H .
$$

For a fixed system of homogeneous coordinates $Z=\left[Z_{0}: Z_{1}: \cdots: Z_{s}\right]$, a hyperplane $H$ of $\mathbb{P}^{s}(\mathbb{C})$ can be written as

$$
H=\left\{\left[Z_{0}: Z_{1}: \cdots: Z_{s}\right] \in \mathbb{P}^{s}(\mathbb{C}) \mid\langle Z, \alpha\rangle=0\right\},
$$

where

$$
\langle Z, \alpha\rangle:=a_{0} Z_{0}+\cdots+a_{s} Z_{s}
$$

and $\alpha=\left(a_{0}, \ldots, a_{s}\right) \in \mathbb{C}^{s+1}$ is a nonzero vector. We write it as

$$
H=\{\langle Z, \alpha\rangle=0\}
$$

for convenience. In particular, we can take $\alpha \in B$, where $B$ is the set of Euclidean unit vectors in $\mathbb{C}^{s+1}$.

Let $H_{1}, \ldots, H_{s+1}$ be hyperplanes in $\mathbb{P}^{s}(\mathbb{C})$. Let $\alpha_{j}=\left(a_{j 0}, \ldots, a_{j s}\right) \in B$ be such that

$$
H_{j}=\left\{\left\langle Z, \alpha_{j}\right\rangle=0\right\}
$$

for $j=1, \ldots, s+1$. Define

$$
D\left(H_{1}, \ldots, H_{s+1}\right):=\left|\operatorname{det}\left(\alpha_{1}^{T}, \ldots, \alpha_{s+1}^{T}\right)\right|
$$

which only depends on $H_{j}$ but does not depend on the choice of $\alpha_{j} \in B$.

Definition 2.1. Let $H_{1}, \ldots, H_{q}$, with $q \geq s+1$, be hyperplanes in $\mathbb{P}^{s}(\mathbb{C})$. Define

$$
D\left(H_{1}, \ldots, H_{q}\right):=\prod_{1 \leq j_{1}<\cdots<j_{s+1} \leq q}\left|\operatorname{det}\left(\alpha_{j_{1}}^{T}, \ldots, \alpha_{j_{s+1}}^{T}\right)\right| .
$$

We say the hyperplane family $H_{1}, \ldots, H_{q}, q \geq s+1$, in $\mathbb{P}^{s}(\mathbb{C})$ is in general position if $D\left(H_{1}, \ldots, H_{q}\right)>0$.

Let $M$ and $N$ be connected Hermitian manifolds of dimension $m$ and $s$ with Hermitian metrics $h_{M}$ and $h_{N}$, respectively. The space $\mathscr{C}(M ; N)$ of continuous mappings between $M$ and $N$ endowed with the compact-open topology is second countable so that a metric can be furnished in $\mathscr{C}(M ; N)$ which induces the compactopen topology.

Remark 2.2. A sequence $\left\{f_{n}\right\}$ in $\mathscr{C}(M ; N)$ converges to $f$ in $\mathscr{C}(M ; N)$ in this topology if and only if $\left\{f_{n}\right\}$ converges to $f$ uniformly on compact subset of $M$.

The space $\mathscr{H}(M ; N)$ of holomorphic mappings from $M$ into $N$ is a closed subspace of $\mathscr{C}(M ; N)$. 
Definition 2.3. A family $\mathscr{F} \subset \mathscr{H}(M ; N)$ is called normal on $M$ if any sequence in $\mathscr{F}$ contains a subsequence which is relatively compact in $\mathscr{H}(M ; N)$, that is, if any sequence $\left\{f_{n}\right\} \subset \mathscr{F}$ contains a subsequence which converges to $f \in \mathscr{H}(M ; N)$ uniformly on every compact subset of $M$.

Throughout this paper, we consider the special case where $M$ is a plane domain and $N$ is a complex projective space.

Let $f: D \rightarrow \mathbb{P}^{s}(\mathbb{C})$ be a holomorphic map and $U$ be an open set in $D$. Any holomorphic mapping $\tilde{f}: U \rightarrow \mathbb{C}^{s+1}$ such that $\rho \circ \tilde{f}(z) \equiv f(z)$ in $U$ is called a representation of $f$ on $U$. For a fixed system of homogeneous coordinates $\left[Z_{0}: Z_{1}: \cdots: Z_{s}\right]$ we set

$$
V_{i}=\left\{\left[Z_{0}: Z_{1}: \cdots: Z_{s}\right] \mid Z_{i} \neq 0\right\}, \quad \text { for } i=0, \ldots, s .
$$

Then every $a \in D$ has a neighborhood $U$ of $a$ such that $f(U) \subset V_{i}$ for some $i$, and $f$ has a representation

$$
\tilde{f}=\left(f_{0}, \ldots, f_{i-1}, 1, f_{i+1}, \ldots, f_{s}\right)
$$

on $U$ with holomorphic functions $f_{0}, \ldots, f_{i-1}, f_{i+1}, \ldots, f_{s}$.

Definition 2.4. For an open subset $U$ of $D$ we call a representation $\tilde{f}=\left(f_{0}, \ldots, f_{s}\right)$ a reduced representation of $f$ on $U$ if $f_{0}, \ldots, f_{s}$ are holomorphic functions on $U$ and have no common zero.

Remark 2.5. Every holomorphic map of $D$ into $\mathbb{P}^{s}(\mathbb{C})$ has a reduced representation on some neighborhood of each point in $D$. Moreover, let $\tilde{f}=\left(f_{0}, \ldots, f_{s}\right)$ be a reduced representation of $f$. For an arbitrary nowhere zero holomorphic function $h,\left(f_{0} h, \ldots, f_{s} h\right)$ is also a reduced representation of $f$. Conversely, for every reduced representation $\left(g_{0}, \ldots, g_{s}\right)$ of $f$, each $g_{i}$ can be written as $g_{i}=h f_{i}$ for some nowhere zero holomorphic function $h$.

Remark 2.6. Every $f \in \mathscr{H}\left(D ; \mathbb{P}^{s}(\mathbb{C})\right)$ has a reduced representation on the totality of $D$ [Fujimoto 1974].

We now give the definition of sharing hyperplanes, which extends the definition of sharing values. Take $f \in \mathscr{H}\left(D ; \mathbb{P}^{s}(\mathbb{C})\right)$. Let $H=\{\langle Z, \alpha\rangle=0\}$ be a hyperplane in $\mathbb{P}^{s}(\mathbb{C})$, where $\alpha=\left(a_{0}, \ldots, a_{s}\right) \in \mathbb{C}^{s+1}-\{0\}$. Let $\tilde{f}=\left(f_{0}, \ldots, f_{s}\right)$ be a reduced representation of $f$. We consider the holomorphic function on $D$

$$
\langle\tilde{f}(z), H\rangle:=a_{0} f_{0}+\cdots+a_{s} f_{s} .
$$

Definition 2.7. Suppose $f, g$ are in $\mathscr{H}\left(D ; \mathbb{P}^{s}(\mathbb{C})\right)$ and $H$ is a hyperplane in $\mathbb{P}^{s}(\mathbb{C})$. If there exist some (thus all) reduced representations of $f$ and $g$ respectively such that $\langle\tilde{f}(z), H\rangle$ and $\langle\tilde{g}(z), H\rangle$ share 0 on $D$, we say that $f$ and $g$ share $H$ on $D$. 
By Remark 2.5, $\langle\tilde{f}(z), H\rangle=0$ is indeed independent of the choice of the reduced representation of $f$. Therefore sharing hyperplanes is well defined.

We will use the notation $\langle f(z), H\rangle$ when some properties are independent of the choice of the reduced representation of $f$. For example, we can say that $\langle f(z), H\rangle$ has finite zeros on $D$.

H. Fujimoto [1974] gave the relation between m-convergence and quasiregularity. In the case of holomorphic maps, we have the following properties. Suppose $\left\{f_{n}\right\} \subset \mathscr{H}\left(D ; \mathbb{P}^{s}(\mathbb{C})\right)$; then $\left\{f_{n}\right\}$ converges uniformly on compact subsets of $D$ to a holomorphic mapping $f$ of $D$ into $\mathbb{P}^{s}(\mathbb{C})$ if and only if, for any $a \in D$, each $f_{n}$ has a reduced representation

$$
\tilde{f}_{n}=\left(f_{n 0}, f_{n 1}, \ldots, f_{n s}\right)
$$

on some fixed neighborhood $U$ of $a$ in $D$ such that $\left\{f_{n i}\right\}$ converges uniformly on compact subsets of $U$ to a holomorphic function $f_{i}$ on $U, i=0,1, \ldots, s$, with the property that

$$
\tilde{f}=\left(f_{0}, f_{1}, \ldots, f_{s}\right)
$$

is a reduced representation of $f$ on $U$.

Main results. Here we shall improve both Theorem $\mathrm{C}$ and Theorem $\mathrm{D}$ and obtain the following results.

Theorem 2.8. Suppose $\mathscr{F} \subset \mathscr{H}\left(D ; \mathbb{P}^{s}(\mathbb{C})\right)$. Let $H_{1}, \ldots, H_{q}$, with $q \geq 2 s+1$, be hyperplanes in $\mathbb{P}^{s}(\mathbb{C})$ located in general position. Suppose that for each $f, g \in \mathscr{F}$, $f$ and $g$ share $H_{j}$ on $D$, for $j=1, \ldots, q$. Then $\mathscr{F}$ is normal on $D$.

Corollary 2.9. Suppose $\mathscr{F} \subset \mathscr{H}\left(D ; \mathbb{P}^{s}(\mathbb{C})\right)$. Let $H_{1}, \ldots, H_{q}$, with $q \geq 2 s+1$, be hyperplanes in $\mathbb{P}^{S}(\mathbb{C})$ located in general position. Suppose that for each $f \in F_{F}$, $f$ omits $H_{j}$ on $D$, for $j=1, \ldots, q$. Then $\mathscr{F}$ is normal on $D$.

Proof. Each $H_{j}(j=1, \ldots, q)$ is a shared value of all $f \in \mathscr{F}$, since $f^{-1}\left(H_{j}\right)=\varnothing$. Thus, the family $\mathscr{F}_{\mathrm{F}}$ satisfies the assumptions of Theorem 2.8 .

Theorem 2.10. Suppose $\mathscr{F}, \mathscr{G} \subset \mathscr{H}\left(D ; \mathbb{P}^{s}(\mathbb{C})\right)$. Let $q \geq 3 s+1$ be a integer, and suppose the following three conditions are satisfied:

(i) For each $f \in \mathscr{F}$, there exist $g \in \mathscr{G}$ and $q$ hyperplanes $H_{1, f}, \ldots, H_{q, f}$ (which may depend on $f$ ) such that $f$ and $g$ share $H_{j, f}$ on $D$, for $j=1, \ldots, q$.

(ii) $\inf \left\{D\left(H_{1, f}, \ldots, H_{q, f}\right): f \in \mathscr{F}\right\}>0$.

(iii) $G$ is normal on $D$.

Then $\mathscr{F}$ is a normal family on $D$.

By Theorem 2.10 we immediately have the following corollary. 
Corollary 2.11. Suppose $\mathscr{F}, \mathscr{G} \subset \mathscr{H}\left(D ; \mathbb{P}^{s}(\mathbb{C})\right)$. Let $H_{1}, \ldots, H_{q}$, with $q \geq 3 s+1$, be hyperplanes in $\mathbb{P}^{s}(\mathbb{C})$ located in general position. Suppose that for each $f \in \mathscr{F}_{F}$

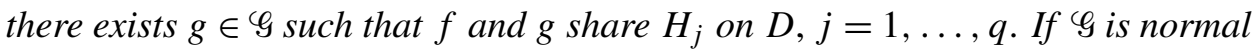
on $D$, then $\mathscr{F}$ is also normal on $D$.

The following example shows that the number $3 s+1$ in Theorem 2.10 is sharp when $s=2$.

Example 1. Let $\Delta$ be the unit disk. Let $\mathscr{F}=\left\{f_{n}(z)\right\}$, where

$$
f_{n}(z)=[\sqrt{-1} \cos n z: \sin n z: \sin n z] .
$$

We denote by $z_{n, 1}, z_{n, 2}, \ldots, z_{n, k_{n}}$ the zeros of $\sin n z$ in $\Delta$. Let $\mathscr{G}=\left\{g_{n}(z)\right\}$, where

$$
g_{n}(z)=\left[1: \prod_{1 \leq i \leq k_{n}} \frac{z-z_{n, i}}{1-\bar{z}_{n, i} z}: \prod_{1 \leq i \leq k_{n}} \frac{z-z_{n, i}}{1-\bar{z}_{n, i} z}\right]
$$

Let

$$
\begin{aligned}
& H_{1}=\left\{\left[Z_{0}: Z_{1}: Z_{2}\right] \mid 3 Z_{0}+Z_{1}+2 Z_{2}=0\right\}, \\
& H_{2}=\left\{\left[Z_{0}: Z_{1}: Z_{2}\right] \mid-5 Z_{0}+Z_{1}+4 Z_{2}=0\right\}, \\
& H_{3}=\left\{\left[Z_{0}: Z_{1}: Z_{2}\right] \mid 7 Z_{0}+Z_{1}+6 Z_{2}=0\right\}, \\
& H_{4}=\left\{\left[Z_{0}: Z_{1}: Z_{2}\right] \mid-9 Z_{0}+Z_{1}+8 Z_{2}=0\right\}, \\
& H_{5}=\left\{\left[Z_{0}: Z_{1}: Z_{2}\right] \mid Z_{2}=0\right\}, \\
& H_{6}=\left\{\left[Z_{0}: Z_{1}: Z_{2}\right] \mid Z_{1}=0\right\} .
\end{aligned}
$$

Then these hyperplanes are in general position.

One can verify that $f_{n}$ and $g_{n}$ share $H_{j}$ on $\Delta$ for $j=1, \ldots, 6$. Clearly, $\varphi$ is normal on $\Delta$. However, F fails to be normal on any neighborhood of 0 by Lemma 3.2 in next section.

\section{Some lemmas}

The following is the general version of the Zalcman lemma.

Lemma 3.1 [Thai et al. 2003]. Let $\mathscr{F}$ be a family of holomorphic mappings of a domain $\Omega$ in $\mathbb{C}^{m}$ into $\mathbb{P}^{s}(\mathbb{C})$. The family $\mathscr{F}$ is not normal on $\Omega$ if and only if there exist sequences $\left\{f_{n}\right\} \subset \mathscr{F},\left\{z_{n}\right\} \subset \Omega$ with $z_{n} \rightarrow z_{0} \in \Omega$, and $\left\{\rho_{n}\right\}$ with $\rho_{n}>0$ and $\rho_{n} \rightarrow 0$ such that

$$
h_{n}(\xi):=f_{n}\left(z_{n}+\rho_{n} \xi\right)
$$

converges uniformly on compact subsets of $\mathbb{C}$ to a nonconstant holomorphic mapping $h$ of $\mathbb{C}$ into $\mathbb{P}^{s}(\mathbb{C})$. 
Lemma 3.2 [Osserman and Ru 1997]. Let $M$ be a Riemann surface, and $f_{n}: M \rightarrow$ $\mathbb{P}^{s}(\mathbb{C})$ be a sequence of holomorphic maps converging uniformly on every compact subset of $M$ to a holomorphic map $f: M \rightarrow \mathbb{P}^{s}(\mathbb{C})$. Given $a, b \in \mathbb{P}^{s}\left(\mathbb{C}^{*}\right)$, let $f_{a, b}$ be the meromorphic function defined by

$$
f_{a, b}=\frac{\langle\tilde{f}, \alpha\rangle}{\langle\tilde{f}, \beta\rangle}
$$

where $\tilde{f}$ is a reduced representation of $f$ on $U$, and $\alpha, \beta \in\left(\mathbb{C}^{s+1}\right)^{*}$ are such that $a=\rho(\alpha), b=\rho(\beta)$. Assume that $\beta(\tilde{f}) \neq 0$ on some $U$. Let $p \in M$ be such that $\beta(\tilde{f})(p) \neq 0$, and $U_{p}$ be a neighborhood of $p$ such that $\beta(\tilde{f})(z) \neq 0$ for $z \in U_{p}$. Then $\left\{f_{n_{a, b}}\right\}$ converges uniformly on $U_{p}$ to the meromorphic function $f_{a, b}$.

Let $\mu>0$ be an integer. The holomorphic map $f \in \mathscr{H}\left(\mathbb{C} ; \mathbb{P}^{s}(\mathbb{C})\right)$ is said to be ramified over a hyperplane $H=\{\langle Z, \alpha\rangle=0\}$ with multiplicity at least $\mu$ if all zeros of $\langle f(z), \alpha\rangle=0$ have orders at least $\mu$, where $\tilde{f}$ is a local reduced representation of $f$ (it is easy to check that this definition is independent of the choice of reduced representation). If either the image of $f$ completely omits $H$ or $f(\mathbb{C}) \subseteq H$, we shall say that $f$ is ramified over $H$ with multiplicity $\infty$.

Nochka [1983] improved the result of Green [1977] and proved H. Cartan's conjecture.

Lemma 3.3 [Nochka 1983]. Suppose that $q(\geq 2 s+1)$ hyperplanes $H_{1}, \ldots, H_{q}$ are given in general position in $\mathbb{P}^{s}(\mathbb{C})$, along with $q$ positive integers $m_{1}, \ldots, m_{q}$ (some of them may be $\infty$ ). If

$$
\sum_{j=1}^{q}\left(1-\frac{s}{m_{j}}\right)>s+1,
$$

then there does not exist a nonconstant holomorphic mapping $f: \mathbb{C} \rightarrow \mathbb{P}^{s}(\mathbb{C})$ such that $f$ intersects $H_{j}$ with multiplicity at least $m_{j}, j=1, \ldots, q$.

Lemma 3.4 (first main theorem [Fujimoto 1993, Corollary 3.1.16]). Let $f: \mathbb{C} \rightarrow$ $\mathbb{P}^{s}(\mathbb{C})$ be a holomorphic map. Let $H$ be a hyperplane in $\mathbb{P}^{s}(\mathbb{C})$. If $f(\mathbb{C}) \nsubseteq H$, then

$$
T_{f}(r)=m_{f}(r, H)+N_{f}(r, H)+O(1) .
$$

The second main theorem about linearly degenerated case is also required.

Lemma 3.5 (degenerate second main theorem [Ru 2001, Theorem A3.4.4]). Let $f=\left[f_{0}: \cdots: f_{s}\right]: \mathbb{C} \rightarrow \mathbb{P}^{s}(\mathbb{C})$ be a holomorphic map whose image is contained in some $k$-dimensional subspace but not in any subspace of dimension lower than $k$. Let $H_{1}, \ldots, H_{q}$ be hyperplanes in general position. Assume that $f(\mathbb{C}) \nsubseteq H_{j}$, for 
$j=1, \ldots, q$. Then the inequality

$$
\sum_{j=1}^{q} m_{f}\left(r, H_{j}\right)+\frac{n+1}{k+1} N\left(R_{f}, r\right) \leq(2 n-k+1) T_{f}(r)+O\left(\log T_{f}(r)\right)
$$

holds for all $r$ outside a set $E$ with finite Lebesgue measure. Here $N\left(R_{f}, r\right)$ is the ramification term.

Lemma 3.6 [Fujimoto 1974]. Let $f \in \mathscr{H}\left(\mathbb{C} ; \mathbb{P}^{s}(\mathbb{C})\right)$. The map $f$ is rational, namely, $f$ is representable as $f=\left[f_{0}: \cdots: f_{s}\right]$ with polynomial $f_{i}, i=0, \ldots, s$, if and only if

$$
\lim _{r \rightarrow \infty} \frac{T_{f}(r)}{\log r}<\infty .
$$

Lemma 3.7. Let $f \in \mathscr{H}\left(\mathbb{C} ; \mathbb{P}^{s}(\mathbb{C})\right)$, and $H_{1}, \ldots, H_{2 s+1}$ be hyperplanes in $\mathbb{P}^{s}(\mathbb{C})$ located in general position. If for each hyperplane $H_{j}, j=1, \ldots, 2 s+1$, either $f(\mathbb{C}) \subset H_{j}$ or $\left\langle f(z), H_{j}\right\rangle$ has finite zeros in $\mathbb{C}$ (no zero point is allowed), then the map $f$ is rational.

Proof. Let $\tilde{f}=\left(f_{0}, \ldots, f_{s}\right)$ be a reduced representation of $f$ on $\mathbb{C}$. We set the rank of the vector group $\left\{f_{0}, \ldots, f_{s}\right\}$ to be $k+1$, with $0 \leq k \leq s$. Thus, $f(\mathbb{C})$ is contained in some $k$-dimensional subspace of $\mathbb{P}^{s}(\mathbb{C})$ but not in any subspace of dimension lower than $k$.

Let $I$ be a subset of $\{1, \ldots, 2 s+1\}$ such that $i$ is in $I$ if and only if $f(\mathbb{C}) \subset H_{i}$, and let

$$
X_{I}=\bigcap_{i \in I} H_{i}
$$

We can identify $X_{I}$ with a projective space of dimension $s-k_{1}$, where $k_{1}=\# I$. So $0 \leq k_{1} \leq s-k$. According to the definition, the restrictions of

$$
H_{j}^{*}:=H_{j} \bigcap X_{I}, \quad j \notin I
$$

are hyperplanes which are still in general position in $X_{I}=\mathbb{P}^{s-k_{1}}(\mathbb{C})$.

Applying Lemma 3.5 to $f=\left[f_{0}: \cdots: f_{s}\right]: \mathbb{C} \rightarrow \mathbb{P}^{s-k_{1}}(\mathbb{C})$ and the hyperplanes $H_{j}^{*}, j \notin I$, and using the first main theorem about holomorphic curves, it follows that the inequality

$$
\left(2 s-k_{1}+1\right) T_{f}(r) \leq \sum_{j \notin I} N_{f}\left(r, H_{j}^{*}\right)+\left(2\left(s-k_{1}\right)-k+1\right) T_{f}(r)+O\left(\log T_{f}(r)\right)
$$

holds for all $r$ outside a set with finite Lebesgue measure. Since $\left\langle f(z), H_{j}^{*}\right\rangle$ has finite zeros in $\mathbb{C}$, this yields the inequality

$$
\left(k_{1}+k\right) T_{f}(r) \leq O\left(\log T_{f}(r)\right)+O(\log r) .
$$


If $k=k_{1}=0$, the rank of the vector group $\left\{f_{0}, \ldots, f_{s}\right\}$ is 1 , which means that $f$ is a constant map.

If $k_{1}+k>0$. Together with Lemma 3.6, the above inequality implies that $f$ is rational. Hence, the lemma is proved.

\section{Proofs of the theorems}

Proof of Theorem 2.8. Fix $g \in \mathscr{F}$. Suppose that $\mathscr{F}$ is not normal on some point $z_{0} \in D$. Suppose there are $k$ hyperplanes $H_{j l}, l=1, \ldots, k$, such that

$$
g\left(z_{0}\right) \in \bigcap_{l=1}^{k} H_{j_{l}} .
$$

Then $k \leq s$. For otherwise $k \geq s+1$, and because $H_{1}, \ldots, H_{q}, q \geq 2 s+1$, are hyperplanes in $\mathbb{P}^{s}(\mathbb{C})$ located in general position, it follows that $g=[0: 0: \cdots: 0]$. This is a contradiction. Therefore, $k \leq s$. Without loss of generality, we assume that there exists a neighborhood $U\left(z_{0}\right) \subset D$ such that for $l=1, \ldots, k_{1}$,

$$
g\left(U\left(z_{0}\right)\right) \subset H_{l},
$$

for $\mu=k_{1}+1, \ldots, k$,

$$
g\left(U\left(z_{0}\right)\right) \cap H_{\mu}=\left\{g\left(z_{0}\right)\right\},
$$

and for $v=k+1, \ldots, 2 s+1$,

$$
g\left(D\left(z_{0}\right)\right) \cap H_{v}=\phi .
$$

In other words, these hyperplanes are divided into three groups.

Observing that normality is a local property, we may suppose that $U\left(z_{0}\right)$ is the unit disk $\Delta$, and $z_{0}=0$. Then by Lemma 3.1 there exist points $z_{n}$ with $z_{n} \rightarrow z_{0} \in D$, positive numbers $\rho_{n}$ with $\rho_{n} \rightarrow 0$, and functions $f_{n} \in \mathscr{F}$ such that

$$
h_{n}(\xi):=f_{n}\left(z_{n}+\rho_{n} \xi\right)
$$

converges uniformly on compact subsets of $\mathbb{C}$ to a nonconstant holomorphic mapping $h$ of $\mathbb{C}$ into $\mathbb{P}^{s}(\mathbb{C})$. Here $\xi \in \mathbb{C}$ satisfies $z_{n}+\rho_{n} \xi \in \Delta$.

We consider two cases.

If $z_{n} / \rho_{n} \rightarrow \infty$, then for each $\xi \in \mathbb{C}, z_{n}+\rho_{n} \xi \neq z_{0}$ when $n$ is large enough. It follows that for $i=k_{1}+1, \ldots, 2 s+1$,

$$
\left\langle f_{n}\left(z_{n}+\rho_{n} \xi\right), H_{i}\right\rangle \neq 0 .
$$

The Hurwitz theorem implies that for $i=k_{1}+1, \ldots, 2 s+1,\left\langle h(\xi), H_{i}\right\rangle \neq 0$ or $\left\langle h(\xi), H_{i}\right\rangle \equiv 0$. Thus, $\left\langle h(\xi), H_{j}\right\rangle \neq 0$ or $\left\langle h(\xi), H_{j}\right\rangle \equiv 0$ for $j=1, \ldots, 2 s+1$. By Lemma $3.3, h$ is a constant holomorphic mapping. This contradicts the claim that 
$h$ is a nonconstant holomorphic mapping.

If $z_{n} / \rho_{n} \nrightarrow \rightarrow \infty$, taking a subsequence and renumbering, we may assume that $z_{n} / \rho_{n} \rightarrow c, c \in \mathbb{C}$. Then

$$
f_{n}\left(\rho_{n} \xi\right)=h_{n}\left(\xi-\frac{z_{n}}{\rho_{n}}\right)
$$

converges uniformly on compact subsets of $\mathbb{C}$ to a nonconstant holomorphic mapping $h(\xi-c)$. Since for each hyperplane $H_{j}, j=1, \ldots, 2 s+1$, either $h(\mathbb{C}) \subset H_{j}$ or $\left\langle h(\xi-c)(z), H_{j}\right\rangle$ has finite zeros in $\mathbb{C}, h(\xi-c)$ is rational by Lemma 3.7. Since $h(\xi-c)$ is a holomorphic mapping, there exist some constants $c_{\nu}$, with $v=k+1, \ldots, 2 s+1$, such that

$$
\left\langle h(\xi-c)(z), H_{\nu}\right\rangle \equiv c_{\nu} .
$$

Note that $2 s-k+1 \geq s+1$, and $\left\{H_{j}\right\}$ are in general position. Hence we see that $h(\xi-c)$ is a constant map. Again, this a contradiction. And hence the family $\mathscr{F}$ is normal on $D$.

Proof of Theorem 2.10. If $\mathscr{F}$ is not normal on $D$, then by Lemma 3.1, there exist points $z_{n} \rightarrow z_{0} \in D$, positive numbers $\rho_{n} \rightarrow 0$ and functions $f_{n} \in \mathscr{F}$, such that

$$
h_{n}(\xi):=f_{n}\left(z_{n}+\rho_{n} \xi\right) \text {, }
$$

where $\xi \in \mathbb{C}$ satisfies $z_{n}+\rho_{n} \xi \in D$, converges uniformly on compact subsets of $\mathbb{C}$ to a nonconstant holomorphic mapping $h$ of $\mathbb{C}$ into $\mathbb{P}^{s}(\mathbb{C})$.

By condition (i), there exist $q$ hyperplane sequences $\left\{H_{j, f_{n}}\right\}_{n=1}^{\infty}$ and $\left\{g_{n}\right\} \subset \mathcal{G}$ such that for $z \in D, j=1, \ldots, q$,

$$
\left\langle g_{n}(z), H_{j, f_{n}}\right\rangle=0
$$

whenever

$$
\left\langle f_{n}(z), H_{j, f_{n}}\right\rangle=0, \quad z \in D .
$$

For $j=1, \ldots, q$, take $\left\{\alpha_{j n}\right\}_{n=1}^{\infty} \subset B$ satisfying

$$
H_{j, f_{n}}=\left\{\left\langle Z, \alpha_{j n}\right\rangle=0\right\} .
$$

Since $B$ is a compact subset of $\mathbb{C}^{s+1}$, there exist $\alpha_{j}=\left(a_{j 0}, \ldots, a_{j s}\right) \in B$ for $j=1, \ldots, q$, and subsequences which (to avoid complication in notation) we again call $\left\{\alpha_{j n}\right\}$ satisfying that $\alpha_{j n} \rightarrow \alpha_{j}$ as $n \rightarrow \infty$. Let

$$
H_{j}=\left\{\left\langle Z, \alpha_{j}\right\rangle=0\right\}
$$

be hyperplanes of $\mathbb{P}^{s}(\mathbb{C}), j=1, \ldots, q$. From condition (i), it follows that

$$
D\left(H_{1}, \ldots, H_{q}\right) \geq \liminf _{n \rightarrow \infty} D\left(H_{1, f_{n}}, \ldots, H_{q, f_{n}}\right)>0 .
$$

Thus, the hyperplanes $H_{j}, j=1, \ldots, q$, are located in general position. 
Claim. There exist at most 2 s hyperplanes such that for each hyperplane $H$, either the image of $h$ completely omits $H$ or $h(\mathbb{C}) \subset H$. If not, Lemma 3.3 shows that $h$ is a constant holomorphic mapping, which is a contradiction. So there exist at least $s+1$ hyperplanes of $H_{j}, j=1, \ldots, q$, such that for $i=1, \ldots, s+1$, $h(\mathbb{C}) \cap H_{i} \neq \phi$ and $h(\mathbb{C}) \nsubseteq H_{i}$.

For a fixed $i \in\{1, \ldots, s+1\}$, suppose that $\xi_{i} \in h(\mathbb{C}) \cap H_{i}$. Choose a small neighborhood $U\left(\xi_{i}\right)$ of $\xi_{i}$ such that $h(\mathbb{C}) \cap H_{i}=\left\{\xi_{i}\right\}$. Hence $\left\langle\tilde{h}\left(\xi_{i}\right), H\right\rangle=0$ and $\left\langle\tilde{h}\left(\xi_{i}\right), H\right\rangle \not \equiv 0$, where $\tilde{h}$ is a local reduced representation. Since $h_{n}$ converges uniformly to $h$ on $U\left(\xi_{i}\right), h_{n}$ has a local reduced representation $\tilde{h}_{n}=\left(h_{n 0}, \ldots, h_{n s}\right)$ such that $\tilde{h}_{n}$ uniformly converges to a reduced representation $\tilde{h}=\left(h_{0}, \ldots, h_{s}\right)$ of $h$ on $U\left(\xi_{i}\right)$. Obviously, $h_{n k}$ converges uniformly to $h_{k}$ on $U\left(\xi_{i}\right)$ for each $k=$ $0, \ldots, s$. Therefore $\left\langle\tilde{h}_{n}(\xi), \alpha_{i n}\right\rangle$ converges uniformly to $\left\langle\tilde{h}(\xi), \alpha_{i}\right\rangle$ on $U\left(\xi_{i}\right)$. By the Hurwitz theorem, there exist $\xi_{i n} \rightarrow \xi_{i}$ such that $\left\langle\tilde{h}_{n}\left(\xi_{i n}\right), \alpha_{i n}\right\rangle=0$, that is, $\left\langle\tilde{f}_{n}\left(z_{n}+\rho_{n} \xi_{i n}\right), \alpha_{i n}\right\rangle=0$.

On the other hand, applying condition (iii), we can find subsequences of $\left\{g_{n}\right\}$ (again denoted by themselves) such that $g_{n}$ converges uniformly to $g$ on $D$, where $g$ is a holomorphic mapping of $D$ into $\mathbb{P}^{s}(\mathbb{C})$. As we noted earlier, $g_{n}$ has a local reduced representation $\tilde{g}_{n}=\left(g_{n 0}, \ldots, g_{n s}\right)$ such that $\tilde{g}_{n}$ uniformly converges to a reduced representation $\tilde{g}=\left(g_{0}, \ldots, g_{s}\right)$ of $g$ on $U\left(z_{0}\right)$. It follows that

$$
\left\langle\tilde{g}_{n}\left(z_{n}+\rho_{n} \xi_{i n}\right), \alpha_{i n}\right\rangle=0 .
$$

As $n \rightarrow \infty$, we have

$$
\left\langle\tilde{g}\left(z_{0}\right), \alpha_{i}\right\rangle=0 .
$$

So there exist $s+1$ hyperplanes $H_{i}, i=1, \ldots, s+1$, which intersect at one point $\rho\left(\tilde{g}\left(z_{0}\right)\right)$. This contradicts the claim that the hyperplanes $H_{j}, j=1, \ldots, q$, are located in general position. This finishes the proof.

\section{Acknowledgements}

The authors thank the referees for valuable suggestions.

\section{References}

[Bergweiler 2006] W. Bergweiler, "Bloch's principle", Comput. Methods Funct. Theory 6:1 (2006), 77-108. MR 2007f:30045 Zbl 1101.30034

[Fujimoto 1974] H. Fujimoto, "On families of meromorphic maps into the complex projective space", Nagoya Math. J. 54 (1974), 21-51. MR 51 \#3543 Zbl 0267.32005

[Fujimoto 1993] H. Fujimoto, Value distribution theory of the Gauss map of minimal surfaces in $\mathbb{R}^{m}$, Aspects of Mathematics E21, Vieweg, Braunschweig, 1993. MR 95d:32029 Zbl 1107.32004

[Green 1977] M. L. Green, "The hyperbolicity of the complement of $2 n+1$ hyperplanes in general position in $\mathbb{P}_{n}$ and related results", Proc. Amer. Math. Soc. 66:1 (1977), 109-113. MR 56 \#15994 Zbl 0366.32013 
[Gundersen 1979] G. G. Gundersen, "Meromorphic functions that share three or four values", $J$. London Math. Soc. (2) 20:3 (1979), 457-466. MR 80m:30030 Zbl 0413.30025

[Liu et al. 2013] X. J. Liu, S. H. Li, and X. Pang, "A normal criterion about two families of meromorphic functions concerning shared values”, Acta Math. Sin. (Engl. Ser.) 29:1 (2013), 151158. MR 3001017 Zbl 1267.30086

[Mai et al. 2005] P. N. Mai, D. D. Thai, and P. N. T. Trang, "Normal families of meromorphic mappings of several complex variables into $\mathbb{P}^{N}(\mathbb{C})$ ", Nagoya Math. J. 180 (2005), 91-110. MR 2006i:32003 Zbl 1162.32001

[Nevanlinna 1926] R. H. Nevanlinna, "Einige Eindueutigkeitssätze in der Theorie der meromorphen Funktionen”, Acta Math. 48 (1926), 367-391.

[Nochka 1983] E. I. Nochka, "On the theory of meromorphic functions", Dokl. Akad. Nauk SSSR 269:3 (1983), 547-552. In Russian; translated in Sov. Math. Dokl. 27 (1983), 377-381. MR 85i:32038 Zbl 0552.32024

[Osserman and Ru 1997] R. Osserman and M. Ru, "An estimate for the Gauss curvature of minimal surfaces in $\mathbb{R}^{m}$ whose Gauss map omits a set of hyperplanes", J. Differential Geom. 46:3 (1997), 578-593. MR 98k:53014 Zbl 0918.53003

[Ru 2001] M. Ru, Nevanlinna theory and its relation to Diophantine approximation, World Scientific, River Edge, NJ, 2001. MR 2002g:11106 Zbl 0998.30030

[Shabat 1985] B. V. Shabat, Distribution of values of holomorphic mappings, Translations of Mathematical Monographs 61, American Mathematical Society, Providence, RI, 1985. MR 86k:32023 Zbl 0564.32016

[Thai et al. 2003] D. D. Thai, P. N. T. Trang, and P. D. Huong, "Families of normal maps in several complex variables and hyperbolicity of complex spaces", Complex Var. Theory Appl. 48:6 (2003), 469-482. MR 2004c:32003 Zbl 1036.32001

[Wu 1967] H. Wu, "Normal families of holomorphic mappings", Acta Math. 119 (1967), 193-233. MR 37 \#468 Zbl 0158.33301

Received August 3, 2013. Revised September 22, 2013.

LIU YANG

DEPARTMENT OF MATHEMATICS

EAST CHINA NORMAL UNIVERSITY

SHANGHAI, 200062

CHINA

yangliu20062006@126.com

CAIYUN FANG

SCHOOL OF MATHEMATICS SCIENCES

NANJING NORMAL UNIVERSITY

NANJING, 210023

CHINA

05325@njnu.edu.cn

\section{Xuecheng Pang}

DEPARTMENT OF MATHEMATICS

EAST CHINA NORMAL UNIVERSITY

SHANGHAI, 200062

CHINA

xcpang@math.ecnu.edu.cn 


\title{
PACIFIC JOURNAL OF MATHEMATICS
}

\author{
msp.org/pjm
}

Founded in 1951 by E. F. Beckenbach (1906-1982) and F. Wolf (1904-1989)

\section{EDITORS}

Don Blasius (Managing Editor)

Department of Mathematics

University of California

Los Angeles, CA 90095-1555

blasius@math.ucla.edu

\author{
Paul Balmer \\ Department of Mathematics \\ University of California \\ Los Angeles, CA 90095-1555 \\ balmer@math.ucla.edu \\ Robert Finn \\ Department of Mathematics \\ Stanford University \\ Stanford, CA 94305-2125 \\ finn@math.stanford.edu \\ Sorin Popa \\ Department of Mathematics \\ University of California \\ Los Angeles, CA 90095-1555 \\ popa@math.ucla.edu
}

\author{
Vyjayanthi Chari \\ Department of Mathematics \\ University of California \\ Riverside, CA 92521-0135 \\ chari@math.ucr.edu \\ Kefeng Liu \\ Department of Mathematics \\ University of California \\ Los Angeles, CA 90095-1555 \\ liu@math.ucla.edu \\ Jie Qing \\ Department of Mathematics \\ University of California \\ Santa Cruz, CA 95064 \\ qing@ cats.ucsc.edu
}

\section{PRODUCTION}

Silvio Levy, Scientific Editor, production@msp.org

\section{SUPPORTING INSTITUTIONS}

ACADEMIA SINICA, TAIPEI

CALIFORNIA INST. OF TECHNOLOGY

INST. DE MATEMÁTICA PURA E APLICADA

KEIO UNIVERSITY

MATH. SCIENCES RESEARCH INSTITUTE

NEW MEXICO STATE UNIV.

OREGON STATE UNIV.

\author{
STANFORD UNIVERSITY \\ UNIV. OF BRITISH COLUMBIA \\ UNIV. OF CALIFORNIA, BERKELEY \\ UNIV. OF CALIFORNIA, DAVIS \\ UNIV. OF CALIFORNIA, LOS ANGELES \\ UNIV. OF CALIFORNIA, RIVERSIDE \\ UNIV. OF CALIFORNIA, SAN DIEGO \\ UNIV. OF CALIF., SANTA BARBARA
}

\author{
Daryl Cooper \\ Department of Mathematics \\ University of California \\ Santa Barbara, CA 93106-3080 \\ cooper@math.ucsb.edu \\ Jiang-Hua Lu \\ Department of Mathematics \\ The University of Hong Kong \\ Pokfulam Rd., Hong Kong \\ jhlu@maths.hku.hk \\ Paul Yang \\ Department of Mathematics \\ Princeton University \\ Princeton NJ 08544-1000 \\ yang@math.princeton.edu
}

These supporting institutions contribute to the cost of publication of this Journal, but they are not owners or publishers and have no responsibility for its contents or policies.

See inside back cover or msp.org/pjm for submission instructions.

The subscription price for 2014 is US $\$ 410 /$ year for the electronic version, and \$535/year for print and electronic.

Subscriptions, requests for back issues and changes of subscribers address should be sent to Pacific Journal of Mathematics, P.O. Box 4163, Berkeley, CA 94704-0163, U.S.A. The Pacific Journal of Mathematics is indexed by Mathematical Reviews, Zentralblatt MATH, PASCAL CNRS Index, Referativnyi Zhurnal, Current Mathematical Publications and Web of Knowledge (Science Citation Index).

The Pacific Journal of Mathematics (ISSN 0030-8730) at the University of California, c/o Department of Mathematics, 798 Evans Hall \#3840, Berkeley, CA 94720-3840, is published twelve times a year. Periodical rate postage paid at Berkeley, CA 94704, and additional mailing offices. POSTMASTER: send address changes to Pacific Journal of Mathematics, P.O. Box 4163, Berkeley, CA 94704-0163.

PJM peer review and production are managed by EditFLOW ${ }^{\circledR}$ from Mathematical Sciences Publishers.

\section{PUBLISHED BY}

\section{mathematical sciences publishers \\ nonprofit scientific publishing}

http://msp.org/

(C) 2014 Mathematical Sciences Publishers 


\section{PACIFIC JOURNAL OF MATHEMATICS}

Volume $272 \quad$ No. $1 \quad$ November 2014

Nonconcordant links with homology cobordant zero-framed surgery $\quad 1$ manifolds

JAE CHOON CHA and MARK POWELL

Certain self-homotopy equivalences on wedge products of Moore spaces

Ho Won CHOI and KeE Young LeE

Modular transformations involving the Mordell integral in Ramanujan's lost notebook

Youn-SEO CHOI

The $D$-topology for diffeological spaces

J. DANiEl Christensen, Gordon SinNAMON and ENXIN Wu

On the Atkin polynomials

AHMAD EL-Guindy and Mourad E. H. ISMAIL

Evolving convex curves to constant-width ones by a perimeter-preserving flow

LAIYUAN GaO and Shengliang PAN

Hilbert series of certain jet schemes of determinantal varieties

SudhiR R. GHORPADE, Boyan Jonov and B. A. SeTHuraman

On a Liu-Yau type inequality for surfaces

Oussama Hijazi, Sebastián Montiel and Simon Raulot

Nonlinear Euler sums

ISTVÁN MEZŐ

Boundary limits for fractional Poisson $a$-extensions of $L^{p}$ boundary functions 227 in a cone

LEI QIAO and TAO ZHAO

Jacobi-Trudi determinants and characters of minimal affinizations

STEVEN V SAM

Normal families of holomorphic mappings into complex projective space concerning shared hyperplanes

LiU Yang, Caiyun Fang and Xuecheng Pang 